\title{
Diffuse pulmonary fibrosis and hiatus hernia
}

\author{
J.E. G. PEARSON and R. S. E. W I L S ON \\ The United Bristol Hospitals and Frenchay Hospital
}

\begin{abstract}
Six patients presenting with initially obscure pulmonary fibrosis are described. Reasons are given for attributing the condition to chronic aspiration pneumonitis from gastro-oesophageal reflux in association with hiatus hernia, supported by lung histology in three cases. Study of a further series of 143 cases of hiatus hernia suggests a $4 \%$ incidence of this insidious complication in addition to any more acute bronchopulmonary episodes. This possibility should be remembered in the differential diagnosis of any patient with pulmonary fibrosis of doubtful origin, as the advanced condition bears a poor prognosis.
\end{abstract}

Although Plummer and Vinson (1921) emphasized that patients with achalasia of the cardia were liable to develop pulmonary complications, the relationship between bronchopulmonary disease and oesophageal dysfunction was not widely recognized until Vinson (1927) explained cause and effect in a case of achalasia complicated by a lung abscess. Aspiration of gastric and oesophageal contents also occurs with pharyngeai diverticulum, carcinoma of the oesophagus or cardia, inflammatory stricture, anaesthesia, and in patients with neurological disorders. Belcher (1949) reviewed 48 patients with dysphagia pneumonitis of whom 10, all with achalasia, had pulmonary fibrosis alone. Anderson, Holman, and Olsen (1953) reviewed 601 cases of achalasia, of whom only three had pulmonary fibrosis as the sole lesion. Belsey (1960) reviewed 1,308 patients with oesophageal disease and found pulmonary complications in a considerable but unspecified number, the commonest being aspiration pneumonitis characterized by haemoptysis, purulent sputum, pleuritic pain, and dyspnoea. Some residual fibrosis or secondary bronchiectasis frequently followed. Other complications included lung abscess, haemoptysis, and collapse. Belsey also commented that progressive pulmonary fibrosis, not associated with an acute episode, was observed 'in a few cases'. However, the number of cases and the associated oesophageal lesion was not stated. Bronchitis, peribronchial infection, pneumonitis, fibrosis of the lower lobes, and aspiration pneumonia are recognized complications of hiatus hernia (Naish and Read, 1965 ; Atkinson, 1967).

In spite of the association of hiatus hernia and lung disease, reviews of pulmonary fibrosis of unknown cause do not consider it as a possible aetiological factor (Scadding, 1956, 1960 ; Herbert, Nahmias, Gaensler, and MacMahon, 1962; Livingstone, Lewis, Reid and Jefferson, 1964; Reid, Cuthbert, and Craik, 1965; Stack, Grant, Irvine, and Moffat, 1965). Castleman and Kibbee (1958) mentioned the presence of a hiatus hernia in a patient dying of idiopathic pulmonary fibrosis but drew no conclusion from this finding.

It is not the intention here to mention the acute or subacute cases of pneumonitis in this condition but to emphasize how the chronic and insidious lesions may create diagnostic difficulty, and to show that they seem to occur with gastrooesophageal reflux from hiatus hernia. By definition the patients in this group are not known to have had any of the acute complications such as lung abscess or infective 'pneumonitis', although these conditions may well lead to chronic disease such as bronchiectasis, which for the same reason is also not considered.

\section{CASE REPORTS}

CASE 1 A 65-year-old housewife sought medical advice in 1960 with heartburn, flatulence, tightness in the chest in the morning, slight dyspnoea, and a dry cough. In 1941 the patient had been investigated for dyspepsia, which in view of a negative barium meal and laparotomy was labelled 'functional dyspepsia'. There was no history of chest disease, arthritis, exposure to occupational dusts or fumes, or allergy, and she was a non-smoker.

On examination slight kyphoscoliosis and scattered crepitations on the right were the only abnormal findings. Her chest radiograph (Fig. 1) suggested scattered fibrosis of the right lung and the left lower lobe. A barium meal revealed a hiatus hernia, and oesophagoscopy showed gastro-oesophageal reflux. 


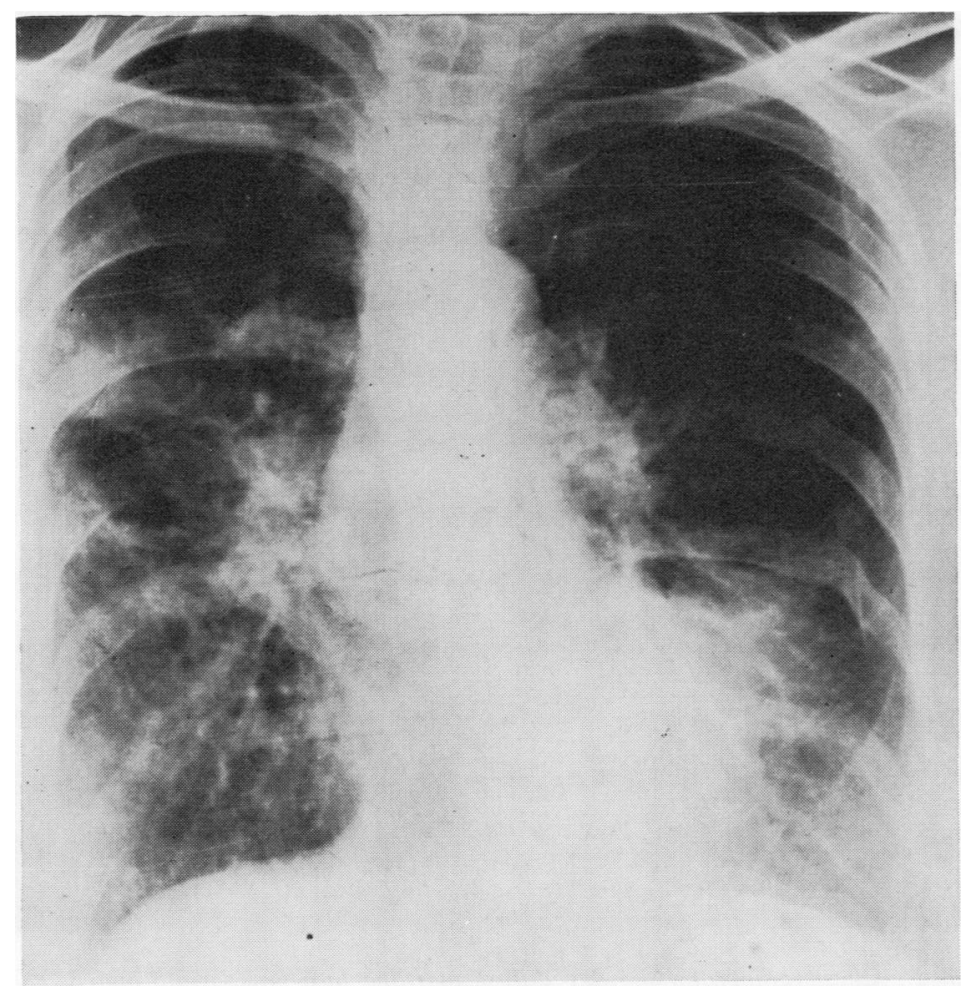

FIG. 1. Chest radiograph of a 65-year-old woman showing scattered lesions in right lung and left lower lobe.

In March 1961 a hiatus hernia repair was performed. This was followed by considerable symptomatic improvement, but by 1967 she was again complaining of dyspepsia and dyspnoea, though by now she was aged 72 .

CASE 2 A 62-year-old wife of a medical practitioner presented with a 10-year history of gastro-oesophageal reflux in 1962. At that time she was noticed to have persistent basal crepitations. A barium meal showed a hiatus hernia, which was repaired in April 1962. In 1969 she complained of an aching pain in the left side of the chest, worse on sitting after exercise. The pain was neither pleuritic nor anginal in type and was thought to be a musculoskeletal disorder due to severe scoliosis and her thoracotomy scar. The patient had noticed increasing dyspnoea and a dry cough over the previous five years, and had suffered from occasional attacks of nocturnal laryngospasm which started 10 years prior to hiatus hernia repair, was absent for two years after operation, and had since recurred. She gave no history of other chest disease, arthritis, exposure to dusts or fumes, or allergy, and was a non-smoker. Examination showed gross scoliosis, with medium crackling sounds over the lower half of both lung fields. A chest radiograph was suggestive of fibrosis of the right lower lobe, with scoliosis obscuring the left side. The electrocardiogram was normal, and a Latex fixation test was negative.

CASE 3 An intelligent 58-year-old businessman was seen as an outpatient in 1959 , with a three-year history of a dry irritating nocturnal cough, relieved by lying on his left side. He confirmed that the cough related to meals and was associated with a congested feeling in the chest, helped by magnesium trisilicate. Because of this a barium meal was performed and showed a hiatus hernia. Oesophagoscopy revealed gastro-oesophageal reflux. He was a non-smoker, and gave no history of acute chest disease, arthritis, allergy or exposure to dusts or fumes.

On examination finger clubbing was present, chest expansion was moderate, and there were scattered bilateral crepitations over the middle and lower portions of the lung. A chest radiograph (Fig. 2) showed diffuse mottling in both lung fields, maximal at the bases. An electrocardiogram was normal, and he was not anaemic. 


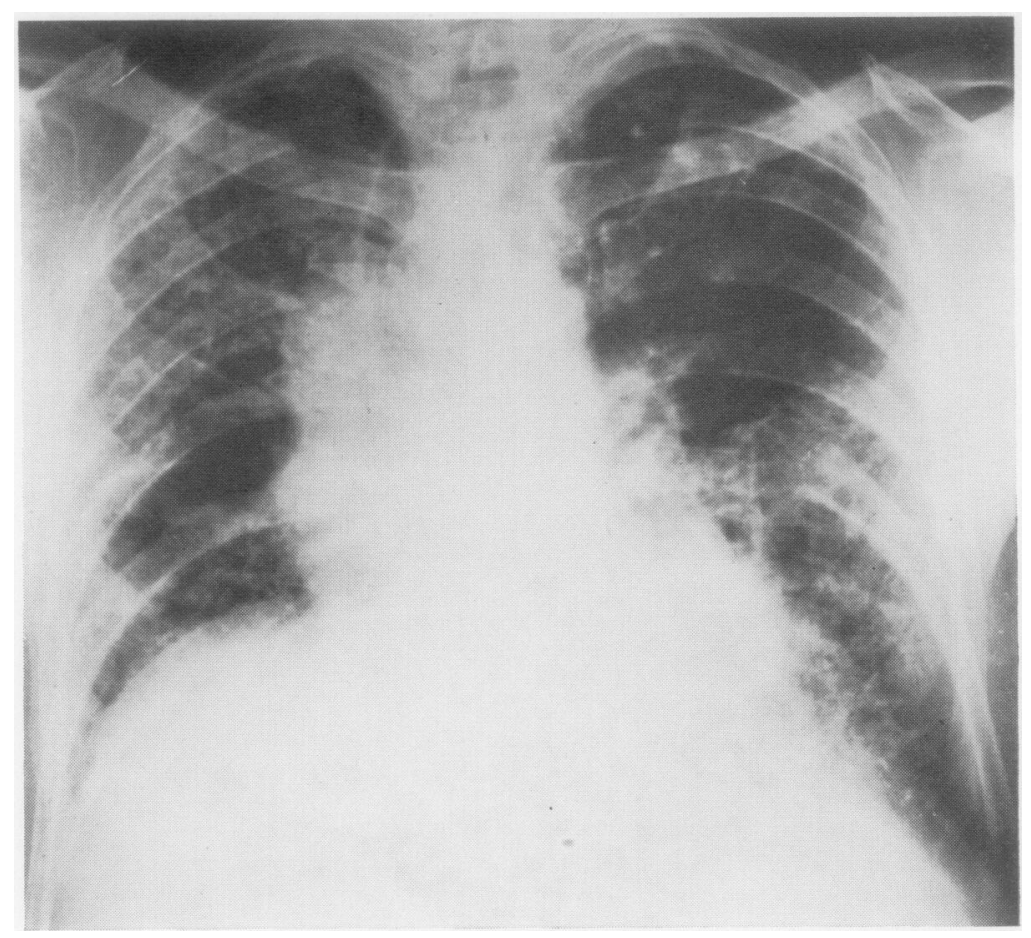

FIG. 2. Chest radiograph showing diffuse mottling in a 58-year-old man.

The hiatus hernia was repaired, and a biopsy was taken from the extensively scarred lungs. Histology showed replacement of the lung tissue by fibrous tissue containing a few lymphocytes. There was alveolar epithelialization with some alveoli containing clusters of macrophages. The pathologist reported 'typical chronic pneumonitis'. The patient's condition slowly deteriorated, and he died of respiratory failure and cor pulmonale within two years. No necropsy was obtained.

CASE 4 A 56-year-old medical practitioner was admitted to hospital with a one-week history of dyspnoea, precordial pain, and weight loss. A history of dyspepsia in 1962 had been attributed to a duodenal ulcer but was mainly one of 'heartburn'. He gave no history of chest disease, allergy, arthritis, or exposure to dusts or fumes. He had smoked 20 cigarettes daily until five years previously.

Examination showed finger clubbing and medium scattered crepitations over both lower lobes. His chest radiograph (Fig. 3) suggested extensive fibrosis and gas transferase (CO uptake by steady state method) was greatly diminished at $6 \mathrm{ml} / \mathrm{mmHg} / \mathrm{min}$ (normal $22-30 \mathrm{ml} / \mathrm{mmHg} / \mathrm{min}$ ). He was not anaemic. Rheumatoid latex fixation test, LE cells in the blood, and examination of the sputum for acid-fast bacilli were negative; an electrocardiogram was normal, and his barium meal showed a hiatus hernia but no duodenal ulceration.

The patient's condition deteriorated rapidly and he died in respiratory failure. At necropsy, the lower end of the oesophagus was dilated, but there was little oesophagitis. The lungs were emphysematous and showed extensive scarring. Histology confirmed widespread irregular areas of interstitial fibrosis, with areas where alveolar spaces were filled with fine fibrous tissue, moderate lymphocyte and macrophage infiltration, and a few plasma cells.

CASE 5 A 60-year-old draughtsman was admitted to hospital for investigation of a 10-year history of dyspnoea, which was worse on exertion. He also complained of a non-productive cough and a nasal discharge. Heartburn and retrosternal pain had been prominent for five years, although a barium meal one year before had revealed no abnormality. He gave no history of arthritis, acute chest disease, allergy, or exposure to dusts or fumes, and had been a nonsmoker for two years.

Examination showed kyphoscoliosis, poor chest $\stackrel{\mathbb{D}}{\circ}$ expansion, and scattered bilateral medium crackling sounds. There was no finger clubbing. His chest radiograph suggested widespread pulmonary fibrosis, 


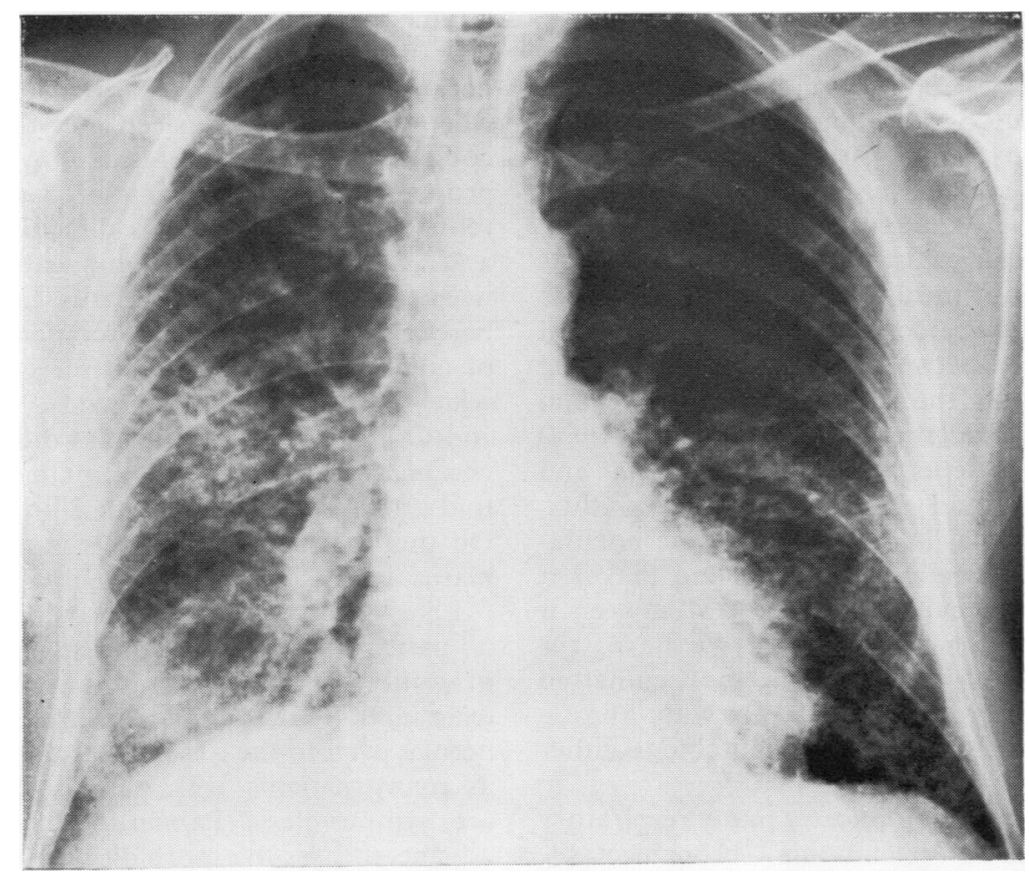

FIG. 3. Chest radiograph of a 56-year-old man showing bilateral mottling, especially in the right.

maximal at the bases. Haemoglobin, white cell count and E.S.R. were within normal limits. Plasma proteins, calcium, and phosphate were normal. Latex fixation test, aspergillus skin tests, Mantoux test (10 T.U.), and examination of the sputum for acid-fast bacilli were negative. The electrocardiogram showed mild right ventricular hypertrophy. Barium meal on this occasion showed a hiatus hernia with reflux. Radiographs of the hands showed no evidence of scleroderma or sarcoidosis. Lung function tests showed a reduced transfer factor at $8.4 \mathrm{ml} / \mathrm{mmHg}$ / $\min$.

In January 1969 a hiatus hernia repair was performed. A lung biopsy taken at that time showed alveolar hyperplasia compatible with chronic inhalation pneumonitis, the cause of which was not apparent: no bronchiectasis was seen. The patient died at home within 18 months of operation and no necropsy was done.

CASE 6 A 74-year-old woman was admitted to hospital with an upper respiratory tract infection, herpes labialis, and widespread crepitations in the lungs, which together with diffuse radiographic shadowing were thought to indicate pneumonia. Her symptoms steadily improved, while the lung signs and radiological appearance did not, so we thought she had an acute infection superimposed on pulmonary fibrosis.
Apart from slight dyspnoea on exertion, her only notable past symptoms had been postural regurgitation and heartburn from the hiatus hernia, diagnosed four years previously and again confirmed by a barium meal. Clinically she was also myxoedematous, but had no arthritis and no finger clubbing. Serum thyroxine was less than $1 \mu \mathrm{g} / 100 \mathrm{ml}$, haemoglobin 9.8 g/100 ml, P.C.V. $31 \%$, M.C.H.C. $32 \%$, rheumatoid latex negative, L.E. cells negative. Spirometry gave FVC 1.6 litres, FEV 1.4 litres $=88 \%$.

The moderate degree of dyspnoea in an anaemic myxoedematous patient with no finger clubbing made it very unlikely that the extensive pulmonary fibrosis was due to fibrosing alveolitis, and, in the absence of other evidence, chronic aspiration pneumonitis seemed the most probable explanation.

\section{REVIEW OF PATIENTS WITH HIATUS HERNIA}

A review of a further 143 consecutive patients with hiatus hernia and gastro-oesophageal reflux revealed six cases with pulmonary symptoms and radiographic changes of chronic pulmonary fibrosis. This is an incidence of about $4 \%$. A further small group of patients showed acute or subacute pulmonary complications. 


\section{DISCUSSION}

Hiatus hernia is a common condition. Cernock (1953) performed barium meal examinations on 200 'normal' people over 50 years old and found three cases of hiatus hernia. Edmunds (1957) and Smellie, Hodson, Waterston, and Astley (1954) estimated the incidence of hiatus hernia in patients undergoing barium meal studies as $3.5 \%$ and $5 \%$ respectively. The incidence in the population at large is probably between $1.5 \%$ and $5 \%$, being more common in the elderly, the obese, and the pregnant. Vandervelde and Carlson (1964) examined 203 patients with hiatus hernia and found that $24 \%$ had gastro-oesophageal reflux. This indicates that a large number of the population have hiatus hernia with reflux and makes it difficult to show a causal relationship between it and chronic pulmonary fibrosis. We have, for instance, met with hiatus hernia in rheumatoid disease and in farmer's lung, both with fibrotic lung changes, which might be attributable to either cause.

Pulmonary fibrosis complicates many respiratory disorders, including pneumonia, bronchiectasis, chronic bronchitis, tuberculosis, atelectasis, irradiation, and the pneumoconioses (Thomson and Cotten, 1962 ; Livingstone et al., 1964). In these conditions the cause of the fibrosis is usually obvious. However, the lesion considered here is a chronic diffuse sometimes patchy fibrosis, in which the cause is not evident. This applied to our six patients, all of whom had hiatus hernia with gastro-oesophageal reflux demonstrated by barium meal and oesophagoscopy.

Aspiration of gastric juice is well recognized. It occurs typically in the patient under anaesthesia and leads to an acute episode after two to four hours, the severity of which depends on the amount and the acidity of the gastric juice inhaled (Mendelson, 1946). It has been shown that small amounts of dilute acid $(5-10 \mathrm{ml}$ of $\mathrm{N} / \mathrm{HCl})$ dropped into the bronchi of cats causes an acute lung lesion with haemorrhagic exudate, total necrosis of the mucous membranes, and eventual fibrosis (Lewiński, 1965).

It is known that hiatus hernia leads to reflux of small amounts of gastric juice containing acid and possibly pepsin. Repeated small aspirations may produce insidious damage to the alveoli and perhaps the terminal bronchioles, leading to widespread fibrosis. There is no infective element because the gastric juice is sterile.

It is difficult to explain how the gastric juice is able to pass the protective mechanism of the larynx without causing any symptoms. Only in case 2 did laryngospasm occur. This may be because the gastic juice is aspirated (Belsey, 1960) when the patient is asleep and the laryngeal and cough reflexes are depressed. Why only a small proportion of the cases of gastro-oesophageal reflux are thus affected is difficult to explain, but a $4 \%$ incidence of this complication is impressive when the frequency of hiatus hernia is remembered. It is interesting to compare the incidence of pulmonary fibrosis in this series of hiatus hernia with that found in rheumatoid arthritis. Stack and Grant (1965) showed that diffuse lung disease was present in only 4 of 117 patients with rheumatoid arthritis, compared with 2 on a control group. On this basis, diffuse fibrosis is more common in hiatus hernia than with rheumatoid disease.

Like Rusby (1963) we would emphasize that all patients with chronic diffuse pulmonary fibrosis of doubtful origin should have a barium meal examination to exclude the presence of a hiatus hernia, even in the absence of a suggestive history, as many patients with gastro-oesophageal reflux are symptomless (Palmer, 1968). In the more advanced cases the morbidity and mortality would seem considerable, half of our patients dying within two years of diagnosis.

\section{REFERENCES}

Anderson, H. A., Holman, C. B., and Olsen, A. M. (1953). Pulmonary complications of cardiospasm. J. Amer. med. Ass., 151, 508.

Atkinson, M. (1967). Hiatus hernia. Brit. med. J., 4, 218.

Belcher, J. R. (1949). The pulmonary complications of dysphagia. Thorax, 4, 44.

Belsey, R. (1960). The pulmonary complications of oesophageal disease. Brit. J. Dis. Chest, 54, 342.

Castleman, B., and Kibbee, B. U. (1958). Case records of the Massachusetts General Hospital. Case 44431. New Engl. J. Med., 259, 830.

Cernock, W. F. (1953). Incidence of asymptomatic hiatus hernia. Amer. J. dig. Dis., 20, 123.

Edmunds, V. (1957). Hiatus hernia. Quart. J. Med., 50 (n.s. 26), 445.

Herbert, F. A., Nahmias, B. B., Gaensler, E. A., and MacMahon, H. E. (1962). Pathophysiology of interstitial pulmonary fibrosis. Arch. intern. Med., 110, 628.

Lewiński, A. (1965). Evaluation of methods employed in the treatment of the chemical pneumonitis of aspiration. Anesthesiology, 26, 37.

Livingstone, J. L., Lewis, J. G., Reid, Lynne, and Jefferson, K. E. (1964). Diffuse interstitial pulmonary fibrosis. Quart. J. Med., 56 (n.s. 33), 71.

Mendelson, C. L. (1946). The aspiration of stomach contents into the lungs during obstetric anesthesia. Amer. J. Obst. Gynec., 52, 191.

Naish, J. M., and Read, A. E. A. (1965). Basic Gastroenterology. Wright, Bristol. 
Palmer, E. D. (1968). The hiatus hernia-esophagitis-esophageal stricture complex. Amer. J. Med., 44, 566.

Plummer, H. S., and Vinson, P. P. (1921). Cardiospasm: a report of 301 cases. Med. Clin. N. Amer., 5, 355.

Reid, J. M., Cuthbert, J., and Craik, J. E. (1965). Chronic diffuse idiopathic fibrosing alveolitis. Brit. J. Dis. Chest, 59, 194.

Rusby, N. L. (1963). In Chest Diseases, vol. 2, edited by K. M. A. Perry and T. H. Sellors, p. 65. Butterworths, London.

Scadding, J. G. (1956). Pulmonary fibrosis and collagen diseases of the lungs: A symposium. 1. Clinical problems of diffuse pulmonary fibrosis. Brit. J. Radiol., 29, 633. (1960). Chronic diffuse interstitial fibrosis of the lungs. Brit. med. J., 1, 443.
Smellie, J. M., Hodson, C. J., Waterston, D. J., and Astley, R. (1954). Discussion on hiatus hernia. Proc. roy. Soc. Med., 47, 531.

Stack, B. H. R., and Grant, I. W. B. (1965). Rheumatoid interstitial lung disease. Brit. J. Dis. Chest, 59, 202.

Irvine, W. J., and Moffat, M. A. J. (1965). Idiopathic diffuse interstitial lung disease. Amer. Rev. resp. Dis., 92, 939.

Thomson, A. D., and Cotton, R. E. (1962). Lecture Notes on Pathology. Blackwell, Oxford.

Vandervelde, G. M., and Carlson, H. C. (1964). Esophageal reflux. Amer. J. Roentgenol., 92, 989.

Vinson, P. P. (1927). Cardiospasm complicated by pulmonary abscess. A case report. Amer. J. Surg., n.s. 2, 359. 